\title{
Image fusion by discrete wavelet transform for multimodal biometric recognition
}

\author{
Arjun Benagatte Channegowda', Hebbakavadi Nanjundaiah Prakash ${ }^{2}$ \\ ${ }^{1}$ Department of Information Science and Engineering, Rajeev Institute of Technology, Hassan, Visvesvaraya Technological University, \\ Belagavi, Karnataka, India \\ ${ }^{2}$ Department of Computer Science and Engineering, Rajeev Institute of Technology, Hassan, Visvesvaraya Technological University,
} Belagavi, Karnataka, India

\begin{tabular}{l} 
Article Info \\
\hline Article history: \\
Received Aug 2, 2021 \\
Revised Oct 17, 2021 \\
Accepted Nov 2, 2021 \\
\hline Keywords: \\
Face \\
Finger vein \\
Image fusion \\
Multimodal biometrics \\
Signature \\
Wavelet transform
\end{tabular}

Wavelet transform

\begin{abstract}
In today's world, security plays a crucial role in almost all applications. Providing security to a huge population is a more challenging task. Biometric security is the key player in such type of situation. Using a biometric-based security system more secure application can be built because it is tough to steal or forge. The unimodal biometric system uses only one biometric modality where some of the limitations will arise. For example, if we use fingerprints due to oiliness or scratches, the finger recognition rate may reduce. In order to overcome the drawbacks of unimodal biometrics, Multimodal biometric systems were introduced. In this paper, new multimodal fusion methods are proposed, where instead of merging features, database images are fused using discrete wavelet transform (DWT) technique. Face and signature images are fused, features are extracted from the fused image, an ensemble classifier is used for classification, and also experiments are conducted for finger vein and signature images.
\end{abstract}

This is an open access article under the $\underline{C C B Y-S A}$ license.

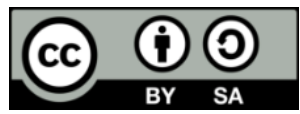

\section{Corresponding Author:}

Arjun Benagatte Channegowda

Department of Information Science and Engineering, Rajeev Institute of Technology, Visvesvaraya

Technological University

Banglore-Manglore highway, Hassan, Karnataka, India

Email: bc.arjun@gmail.com

\section{INTRODUCTION}

The multimodal biometric systems give better results when compared to unimodal biometric systems. In multimodal biometric, more information is available compared to unimodal biometric. More than one biometric information is available in multimodal biometrics [1]-[3]. But designing the multimodal biometric system is a challenging task since to fuse the information of more than one biometric modalities, there will be a lot of compatibility issues. If two biometric modalities features are fused, then there may be chances of unwanted features stored as a template, or most important data may lose due to feature compatibility like data types. To overcome such limitations in multimodal biometrics raw image fusion is used, in the literature [4]-[6] we observed that discrete wavelet transform (DWT) image fusion techniques is not used on multimodal biometric recognition. The biometric fusion at the feature and sensor level is noticeable from the many research work [7]-[10]. In this paper new model is proposed where without extracting the features, raw images are fused using the discrete wavelet transform method, and experiments are conducted on Histogram oriented gradient and uniform local binary pattern features [11]. In discrete wavelets, transform images are decomposed into different levels, and new coefficients are generated. This 
co-efficient is combined or merged to form a new co-efficient that holds the information of both images [12]. And in the next stage, inverse -discrete wavelet transform is applied to get back the fused image.

\section{RESEARCH METHODOLOGY}

In discrete wavelets transform, images are fused by converting them into wavelets, as shown in Figure 1. In order to fuse the images, more than one image is required. These images can be either with the same resolution or a different resolution. Two different methods are used to fuse, as shown in Figures 1(a) and (b).

Figure 1(a) is used to fuse the images of the same resolution, and Figure 1(b) is used to fuse the image with a different resolution [12]. In the initial stage of sampling and registration, the technique is applied to make sure; images are of the same size and registered to the same sensor. After completion of sampling and registration process DWT is applied to images in order to generate coefficient values for very individual pixel of input image. These coefficients are merged to form new coefficient values. In the next step, inverse DWT is applied to a new co-efficient to obtain the fused image. The information of both input images and present in final fused image. Figure 1(a) shows the transformation of image fusion using wavelets for the same resolution image, and Figure 1(b) shows the transformation of image fusion using wavelets for different resolution images. The top left corner shows the levels of decomposition of an image [6], [12].

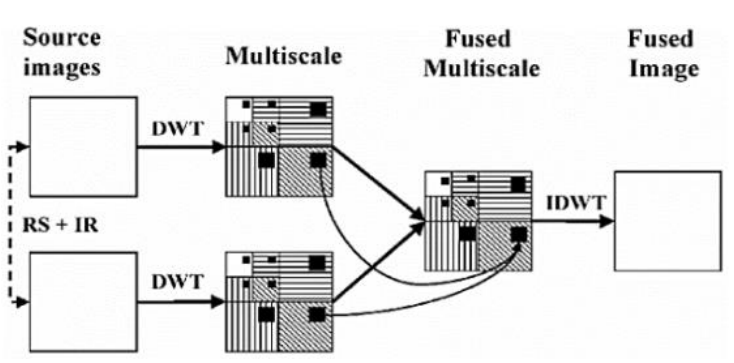

(a)

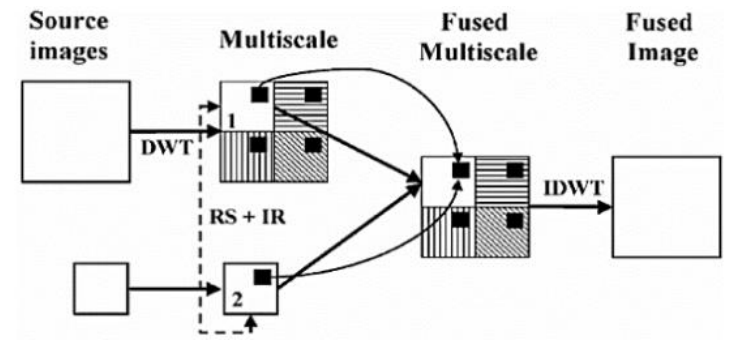

(b)

Figure 1. Discrete wavelets transform for (a) method 1 and (b) method 2

\section{MULTI-RESOLUTION DISCRETE WAVELET TRANSFORM (DWT) FOR PROPOSED WORK}

This paper used multi-resolution wavelet fusion, face, signature, and finger vein images with different resolution images to combine. We used a combination of face and signature also finger vein and signature for experiments. Consider a face sample in multi-resolution wavelet transform shown in Figure 2, shows the stage 1 wavelet transform and Figure 3 shows the $1^{\text {st }}, 2^{\text {nd }}$ and $3^{\text {rd }}$ degree decomposition levels of face image where low pass and high pass filters are applied for each row and column, and four sub-images are created. These four images represent the different decomposed degrees [6], [12].

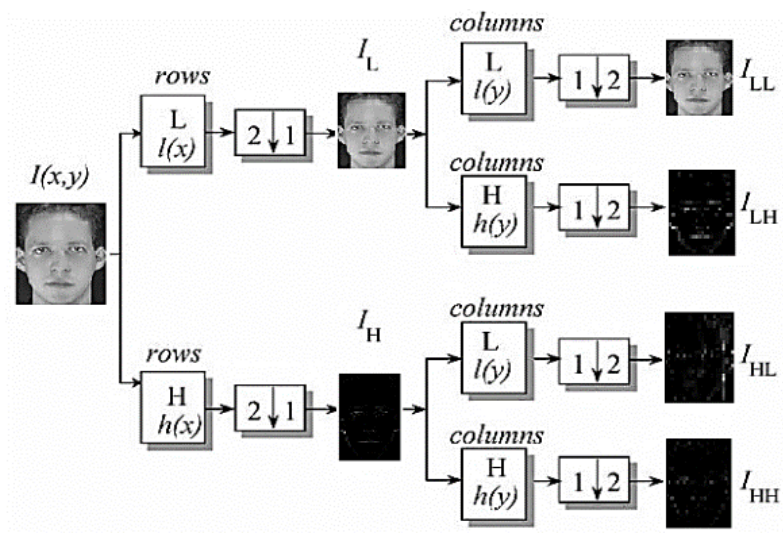

Figure 2. Stage 1 wavelet transform 

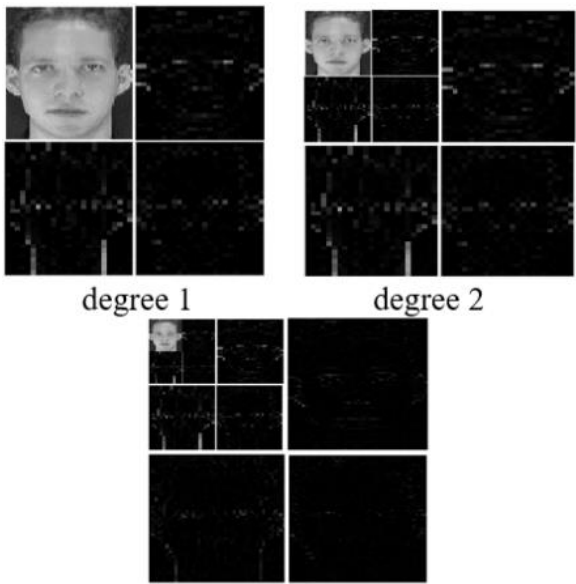

degree 3

Figure 3. First, second, and third degree decomposition levels

After stage 1, with the 3-degree decomposition of the face image, Figure 3 shows the details of 3 degree wavelet decomposition, where the top left corner shows the decomposition degrees. After applying stage 1 transform, the inverse transformation of wavelet is applied to co-efficient, which are obtained as the output of stage 1. In stage 2, inverse DWT is applied, and the final merged image is obtained. The detailed steps are showed in Figure 4. Figure 5 gives the detailed steps of stage 1 and stage 2 transformations with notations.

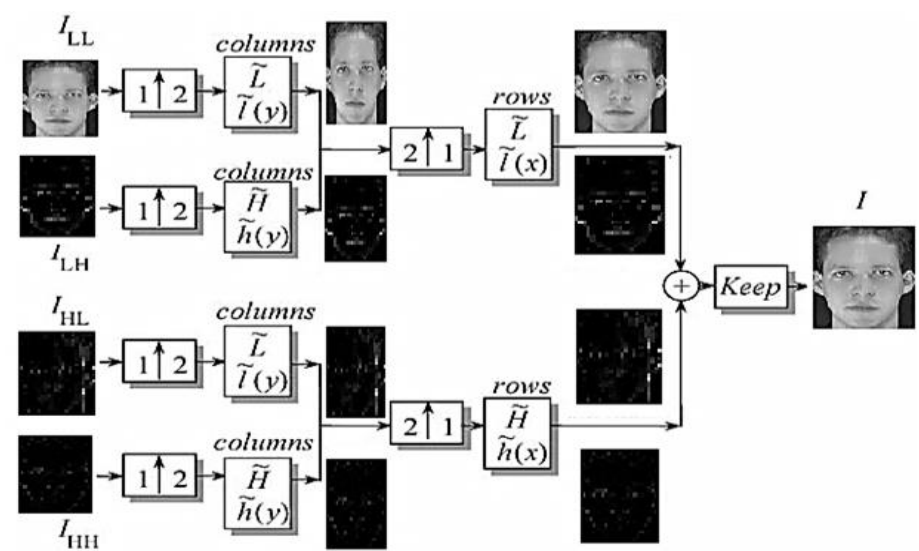

Figure 4. Stage 2 wavelet transform

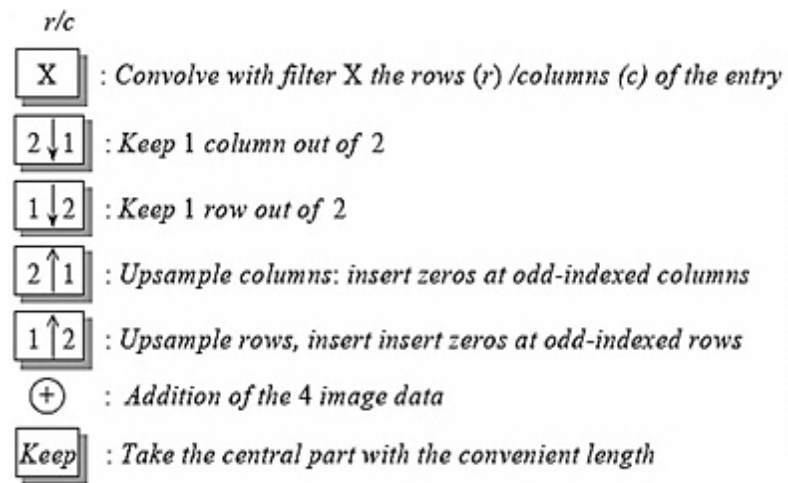

Figure 5. Steps of stage 1 and stage 2 wavelet transformation 


\section{DATABASE USED FOR EXPERIMENTATION}

- Face database: american telephone and telegraph company (AT and T) laboratories Cambridge face database [13] is used, 40 persons, six samples of each individual is used for experimentations.

- Finger vein database: machine learning and data-mining lab, Shandong University (SDUMLA) finger vein database [14] is used. Six samples of the left index finger of the first 40 subjects are used for experimentations.

- Signature database: biometric research lab (ATVS) Madrid Spain's MCYT signature database [13] is used. In this paper, we considered the first 40 subjects/individuals with the first six genuine samples of each individual. Finally 720 samples of face, signature and finger vein are used for experimentation. The samples are mapped one to one for forty individuals. Mapping of data base is commonly used by all the researchers due to non availability of standard data bases, and also it has been showed that the performance of the fusion models will be same when compared with mapped data base and original data base of same person. Figure 6 shows the image samples of MCYT face, SDUMLA finger vein, and MCYT signature is shown in Figure 6(a), Figure 6(b), and Figure 6(c) respectively.

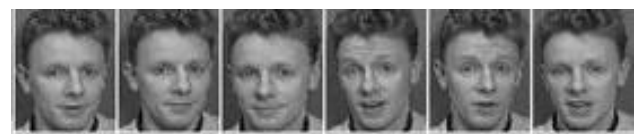

(a)
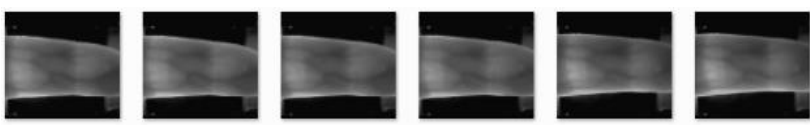
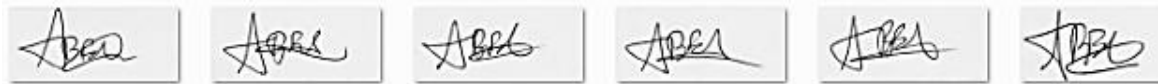

(c)

Figure 6. Image samples for (a) MCYT face, (b) SDUMLA finger vein, and (c) MCYT signature

\section{PROPOSED IMAGE FUSION TECHNIQUE}

In this paper, research work is carried out to increase the recognition rate of multimodal systems over unimodal systems by applying the wavelet fusion technique to multimodal biometrics. In the proposed method, we fused face and signature images directly by fetching the images from the standard database. Experiments are also explored on finger vein and signature database. The fusion of images using DWT for biometric recognition for the face with signature and finger vein with signature for the standard database is a novel approach.

\subsection{Fusion of face images and signature images}

In the proposed work, standard data samples or images of face and signature are fused using DWT, 240 samples of signatures are fused with 240 samples of face one to one, and new data samples are created. This new database with fused images has information from both signature and face biometric samples. The Figure 7 shows fusion of face and signature and Figure 8 shows the fusion of Finger vein and signature by using both DWT stage 1 and IDWT stage 2 of Figure 2 and Figure 4. The final output image contains a rich information source than the original images.

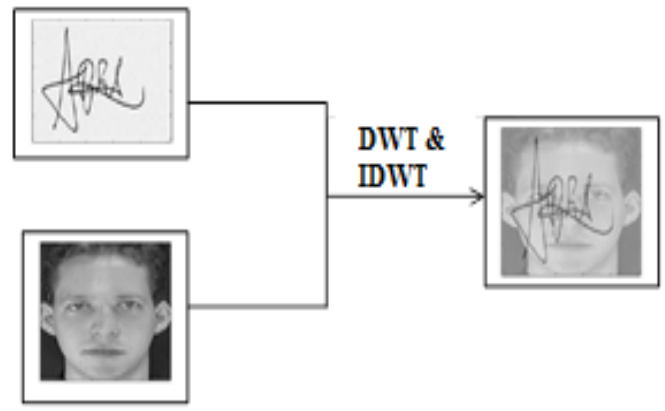

Figure 7. Wavelet fusion face signature

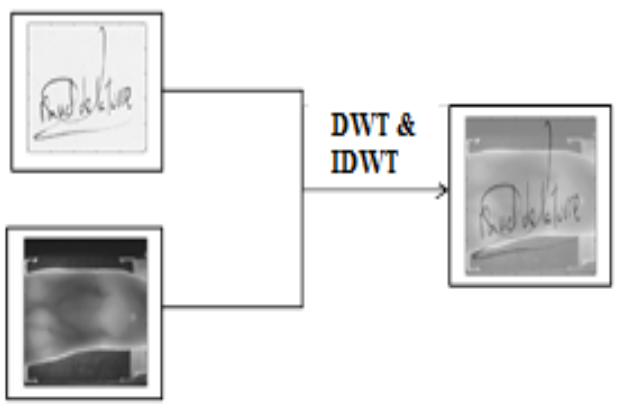

Figure 8 . Wavelet fusion finger vein signature 


\subsection{Fusion of finger vein images and signature images}

In the proposed work, standard data samples of finger vein and signature are fused using DWT, 240 samples of signatures are fused with 240 samples of finger vein one to one, and new data samples are created. This new database with fused images has information from both signature and finger vein biometric samples. Figure 8 shows the fusion of finger vein and signature by using both DWT stage 1 and IDWT stage 2 of Figure 2 and Figure 4. The final output image contains a rich information source than the original images.

\subsection{Fusion of finger vein, signature and face}

In the proposed work, standard data samples of finger vein, signature and face are fused using DWT, 240 samples of signatures are fused with 240 samples of finger vein and 240 samples of face one to one, and new data samples are created. This new database with fused images has information from signature, finger vein and face biometric samples. Figure 9 shows the fusion of finger vein, signature and face by using both DWT stage 1 and IDWT stage 2 of Figure 2 and Figure 4 . The final output image contains a rich information source than the original images.

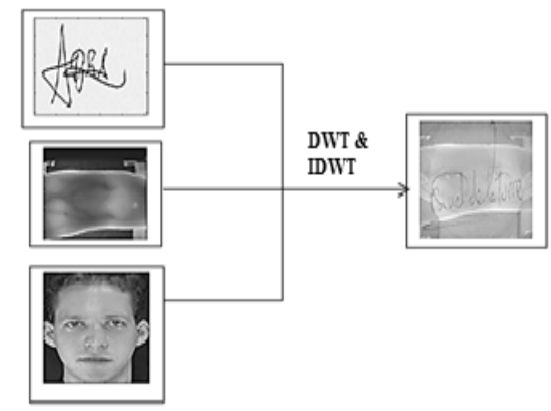

Figure 9. Wavelet fusion finger vein, face, and signature

\section{PROPOSED FUSION MODEL}

In this paper, image fusion is applied using the wavelet transformation technique, there are many wavelet filters available, and from the literature [15]-[20], it has been observed that the db2 filter is best suited for multi-resolution images. In this paper, we used the db2 wavelet filter for transformation. And it has been identified in the literature that more decomposition is loose the details and less decomposition will overlap; hence degree 5 decompositions is used for our work. We explored the experiments by combining face with signature and finger vein with the signature and finally combining all the three biometrics. All three multimodal biometric recognition models were explored in this paper. Figure 10 shows the proposed model for face, signature and finger vein biometric samples, and the same method is used to implement the face and finger vein model and face and signature model.

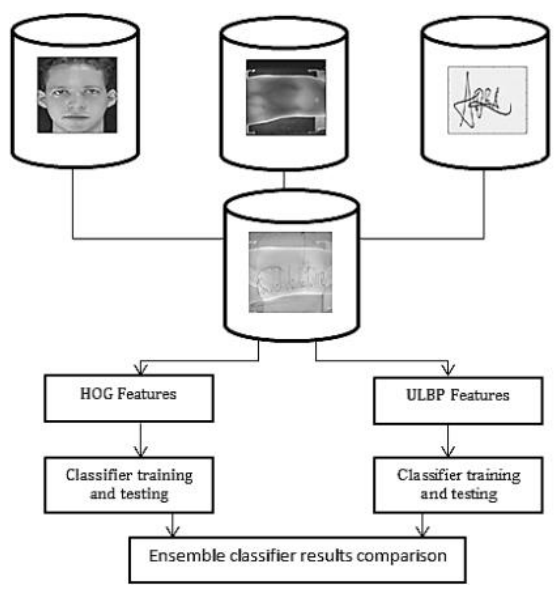

Figure 10. Proposed DWT fusion model 


\section{EXPERIMENTS AND RESULTS}

We identified that image fusion using DWT is used for image enhancement in most of the research work from the literature. In this paper, we used the DWT image fusion technique for multimodal biometric recognition. And in multimodal biometric, not much work is explored on face and signature combination. As per our knowledge, combinations of finger vein and signature with DWT image fusion are novel approaches. All the experiments are conducted on standard databases with larger size data samples. Three experiments are conducted on the proposed model. First experiments on the face and signature biometric modalities were explored. And in the next stage, finger vein and signature biometric modalities have experimented and in last stage face, finger vein and signature are experimented. For the biometric samples of face, signature and finger vein, both unimodal and multimodal biometric investigation is conducted. I to XII represent the experiments in Tables 1 to 5 .

\subsection{Face and signature fusion experiments}

Separate unimodal experiments are conducted on face and signature using uniform local binary pattern (ULBP) features and histogram of oriented gradient (HOG) features [21]-[26]. In the entire case, an ensemble machine learning classifier is used [26]. Unimodal experiment results are shown in Table 1.

Table 1. Unimodal biometric experiments

\begin{tabular}{ccc}
\hline Unimodal & Biometric & Accuracy of Classifier \\
\hline I & Face (ULBP) & $74 \%$ \\
II & Face (HOG) & $82 \%$ \\
III & Signature (ULBP) & $85 \%$ \\
IV & Signature (HOG) & $91 \%$ \\
\hline
\end{tabular}

In multimodal experiments, fused samples of face and signature are used. From the fused images, ULBP and HOG features are extracted. An ensemble classifier is used for classification [25], [26]. Multimodal experiment results are shown in Table 2.

Table 2. Multimodal experiments

\begin{tabular}{ccc}
\hline Multimodal & Biometric & Accuracy of Classifier \\
\hline V & Face Signature (ULBP) & $92 \%$ \\
VI & Face Signature (HOG) & $94 \%$ \\
\hline
\end{tabular}

\subsection{Finger vein and signature fusion experiments}

Separate unimodal experiments are conducted on finger vein and signature using ULBP features and HOG features. In the entire case ensemble, a machine learning classifier is used [25], [26]. Unimodal experiments result shown in Table 3.

Table 3. Unimodal biometric experiments

\begin{tabular}{ccc}
\hline Unimodal & Biometric & Accuracy of Classifier \\
\hline VII & Finger vein (ULBP) & $88 \%$ \\
VIII & Finger vein (HOG) & $82 \%$ \\
\hline
\end{tabular}

In multimodal experiments, fused samples of finger vein and signatures are used, from the fused images ULBP, and HOG features are extracted. An ensemble classifier is used for classification [26]. Multimodal experiments result shown in Table 4. Overall, twelve experiments are conducted in which proposed multimodal experiments outperforms over unimodal experiments.

Table 4. Multimodal experiments

\begin{tabular}{ccc}
\hline Multimodal & Biometric & Accuracy of Classifier \\
\hline IX & Finger vein Signature (ULBP) & $94 \%$ \\
X & Finger vein Signature (HOG) & $97 \%$ \\
\hline
\end{tabular}




\subsection{Finger vein, face, and signature fusion experiments}

After experimenting the combinations of face-signature and finger vein-signature, All the three biometrics are fused together to conduct new experiment, from the fused images ULBP, and HOG features are extracted. An ensemble classifier is used for classification [26]. Multimodal experiments result shown in Table 5.

Table 5. Multimodal experiments

\begin{tabular}{ccc}
\hline Multimodal & Biometric & Accuracy of Classifier \\
\hline XI & Finger vein, Face, and Signature (ULBP) & $92.5 \%$ \\
XII & Finger vein, Face, and Signature (HOG) & $95.8 \%$ \\
\hline
\end{tabular}

\subsection{Comparision of results with previous research works}

From the literature survey of the previous work [27]-[36], it has been observed that, very limited work is investigated by the researchers previously on the wavelet fusion for multimodal biometric recognition. The pervious work on finger vein and signature combination is done on cryptography [37], in which the author used only one sample for authentication. The pervious works were not explored on multimodal biometric recognitions. The standard database with larger data samples is not considered in the previous work. In this paper, we explored the work on larger standard databases for face, finger vein and signature combinations. We used 720 data samples of face, signature and finger vein. The Table 6 shows the performance of some latest multimodal biometric research work compared with the proposed system.

Table 6. Comparison with state of art work

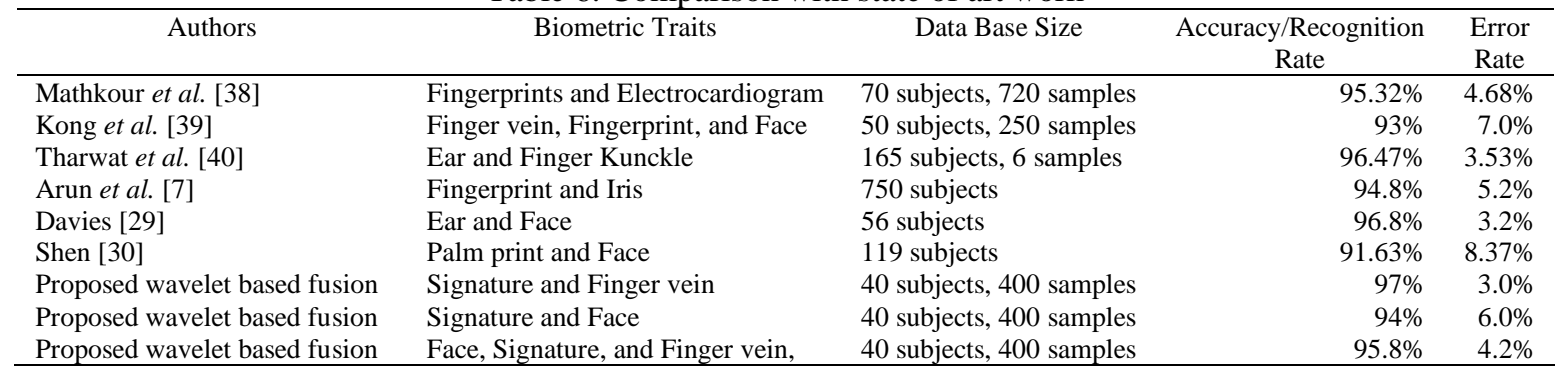

The proposed method significantly reduced the storage space of the image samples when it is compared with the other state of art work. The combinations of Finger vein and signature using wavelet fusion technique shows significant reduction in error rate when compared with other state of art work shown in Table 6. The wavelet based fusion merges the two images of a different biometric characteristics of a person to single image, which significantly reduce the storage space. The proposed technique also showed the features extraction technique ULBP and HOG can be used to improve the recognition rates. The feature extraction techniques ULBP and HOG give the statistical features of the complete image. In the proposed work complete statistical information of samples is used, which is not done in the previous state of art work. The proposed multimodal biometric authentication schemes showed promising results and improved the classification performance rates, when considered recognition rate. With the comparison of pervious work in Table 6 it has been observed that the proposed model showed promising results in improving the biometric recognition rate.

\section{CONCLUSION}

In this paper, we proposed the image fusion technique for multimodal biometric using wavelet transform. We introduced a new model where biometric images are fused using DWT, and these fused images can be used for recognition using machine learning algorithms by extracting HOG and ULBP features. We conducted experiments on the face with signature and finger vein with the signature, and face, signature and finger vein combinations. The fusion combinations of the proposed model, performed well over the unimodal biometric system. Both unimodal and multimodal experiments are conducted, and results are plotted. Twelve experiments are conducted on standard mapped databases. After investigation of all the experiments, it has been observed that wavelet-based image fusion with $\mathrm{db} 2$ wavelet filter and decomposition degree at 5 shows a better recognition rate in finger vein and signature for the selected database. And also 
Histogram oriented gradient features showed better results over uniform local binary patterns in DWT image fusion for multimodal biometrics.

\section{REFERENCES}

[1] A. K. Jain, A. Ross, and S. Prabhakar, "An introduction to biometric recognition," IEEE Transactions on Circuits and Systems for Video Technology, vol. 14, no. 1, pp. 4-20, 2004, doi: 10.1109/TCSVT.2003.818349.

[2] A. Ross, "An introduction to multibiometrics," in European Signal Processing Conference, 2007, pp. 20-24, doi: 10.1007/978-0387-71041-9_14.

[3] A. Ross and A. Jain, "Information fusion in biometrics," Pattern Recognition Letters, vol. 24, no. 13, pp. 2115-2125, 2003, doi: 10.1016/S0167-8655(03)00079-5.

[4] K. Amolins, Y. Zhang, and P. Dare, "Wavelet based image fusion techniques - An introduction, review and comparison," ISPRS Journal of Photogrammetry and Remote Sensing, vol. 62, no. 4. pp. 249-263, 2007, doi: 10.1016/j.isprsjprs.2007.05.009.

[5] M. K. Bhowmik, D. Bhattacharjee, M. Nasipuri, D. K. Basu, and M. Kundu, "Fusion of wavelet coefficients from visual and thermal face images for human face recognition - a comparative study," International Journal of Image Processing, vol. 4, no. 1, pp. 12-23, Jul. 2010, [Online]. Available: http://arxiv.org/abs/1007.0626.

[6] B. Jiang et al., "A comparative study of wavelet-based image fusion with a novel fusion rule II," in Proceedings - 5th International Conference on Instrumentation and Measurement, Computer, Communication, and Control, IMCCC 2015, 2016, pp. 1304-1309, doi: 10.1109/IMCCC.2015.280.

[7] A. A. Ross, K. Nandakumar, and A. K. Jain, Handbook of multibiometrics, 1st ed., vol. 6. Boston: Springer Science and Business Media, 2006.

[8] M. O. Oloyede and G. P. Hancke, "Unimodal and multimodal biometric sensing systems: a review," IEEE Access, vol. 4. pp. 7532-7555, 2016, doi: 10.1109/ACCESS.2016.2614720.

[9] A. Ross and A. K. Janil, "Multimodal biometrics: An overview," in 12th European Signal Processing Conference, 2004, pp. 1221-1224.

[10] M. I. Razzak, R. Yusof, and M. Khalid, "Multimodal face and finger veins biometric authentication," Scientific Research and Essays, vol. 5, no. 17, pp. 2529-2534, 2010.

[11] P. J. H. Prof. Matti Pietikäinen, "Image and video description with local binary pattern variants," SpringerBriefs in Applied Sciences and Technology, 2016.

[12] G. Pajares and J. Manuel de la Cruz, “A wavelet-based image fusion tutorial,” Pattern Recognition, vol. 37, no. 9, pp. 1855-1872, Sep. 2004, doi: 10.1016/j.patcog.2004.03.010.

[13] J. Ortega-Garcia et al., "MCYT baseline corpus: a bimodal biometric database," IEE Proceedings: Vision, Image and Signal Processing, vol. 150, no. 6, pp. 395-401, 2003, doi: 10.1049/ip-vis:20031078.

[14] Y. Yin, L. Liu, and X. Sun, "SDUMLA-HMT: a multimodal biometric database," in Lecture Notes in Computer Science (including subseries Lecture Notes in Artificial Intelligence and Lecture Notes in Bioinformatics), vol. 7098 LNCS, 2011, pp. 260-268.

[15] D. Suresha and H. N. Prakash, "Single picture super resolution of natural images using N-Neighbor Adaptive Bilinear Interpolation and absolute asymmetry based wavelet hard thresholding," in Proceedings of the 2016 2nd International Conference on Applied and Theoretical Computing and Communication Technology, iCATccT 2016, 2017, pp. 387-393, doi: 10.1109/ICATCCT.2016.7912029.

[16] M. Tico, E. Immonen, P. Ramo, P. Kuosmanen, and J. Saarinen, "Fingerprint recognition using wavelet features," in ISCAS 2001 - 2001 IEEE International Symposium on Circuits and Systems, Conference Proceedings, 2001, vol. 2, pp. 21-24, doi: 10.1109/ISCAS.2001.920996.

[17] B. Aiazzi, L. Alparone, S. Baronti, and A. Garzelli, "Context-driven fusion of high spatial and spectral resolution images based on oversampled multiresolution analysis," IEEE Transactions on Geoscience and Remote Sensing, vol. 40, no. 10, pp. 2300-2312, 2002, doi: 10.1109/TGRS.2002.803623.

[18] N. S. Kumar and C. Shanthi, "A survey and analysis of pixel level multisensor medical image fusion using discrete wavelet transform," IETE Technical Review (Institution of Electronics and Telecommunication Engineers, India), vol. 24, no. 2, pp. 113125, 2007, doi: 10.4103/02564602.10876590.

[19] L. Prasad and S. S. Iyengar, Wavelet analysis with applications to image processing, 1st ed. Boca Raton: CRC Press, 2020.

[20] H. Kaur, D. Koundal, and V. Kadyan, "Multi modal image fusion: comparative analysis," in Proceedings of the 2019 IEEE International Conference on Communication and Signal Processing, ICCSP 2019, 2019, pp. 758-761, doi: 10.1109/ICCSP.2019.8697967.

[21] A. B. Channegowda and H. N. Prakash, "Multimodal biometrics of fingerprint and signature recognition using multi-level feature fusion and deep learning techniques," Indonesian Journal of Electrical Engineering and Computer Science, vol. 22, no. 1, p. 187, 2021, doi: 10.11591/ijeecs.v22.i1.pp187-195.

[22] Z. Rustam, Y. Amalia, S. Hartini, and G. S. Saragih, "Linear discriminant analysis and support vector machines for classifying breast cancer," IAES International Journal of Artificial Intelligence, vol. 10, no. 1, pp. 253-256, 2021, doi: 10.11591/ijai.v10.i1.pp253-256.

[23] A. Ramachandran, A. Ramesh, A. Sukhlecha, A. Pandey, and A. Karuppiah, "Machine learning algorithms for fall detection using kinematic and heart rate parameters-a comprehensive analysis," IAES International Journal of Artificial Intelligence, vol. 9, no. 4, pp. 772-780, 2020, doi: 10.11591/ijai.v9.i4.pp772-780.

[24] N. Dalal and B. Triggs, "Histograms of oriented gradients for human detection," in Proceedings - 2005 IEEE Computer Society Conference on Computer Vision and Pattern Recognition, CVPR 2005, 2005, vol. I, pp. 886-893, doi: 10.1109/CVPR.2005.177.

[25] B. C. Arjun and H. N. Prakash, "Multimodal biometric recognition: fusion of modified adaptive bilinear interpolation data samples of face and signature using local binary pattern features," International Journal of Engineering and Advanced Technology, vol. 9, no. 3, pp. 3111-3120, Feb. 2020, doi: 10.35940/ijeat.C6117.029320029320.

[26] B. C. Arjun and H. N. Prakash, "Feature level fusion of seven neighbor bilinear interpolation data sets of finger vein and iris for multimodal biometric recognition," International Journal of Advanced Trends in Computer Science and Engineering, vol. 9, no. 2, pp. 1531-1536, 2020, doi: 10.30534/ijatcse/2020/95922020.

[27] K. Gupta, "Advances in multi modal biometric systems: a brief review," in Proceeding - IEEE International Conference on Computing, Communication and Automation, ICCCA 2017, 2017, vol. 2017-January, pp. 262-267, doi: 
10.1109/CCAA.2017.8229811.

[28] M. Ghayoumi, "A review of multimodal biometric systems: fusion methods and their applications," in 2015 IEEE/ACIS 14th International Conference on Computer and Information Science, ICIS 2015 - Proceedings, 2015, pp. 131-136, doi: 10.1109/ICIS.2015.7166582

[29] S. M. S. Islam, R. Davies, M. Bennamoun, R. A. Owens, and A. S. Mian, "Multibiometric human recognition using 3D ear and face features," Pattern Recognition, vol. 46, no. 3, pp. 613-627, 2013, doi: 10.1016/j.patcog.2012.09.016.

[30] L. Shen, L. Bai, and Z. Ji, "FPCode: an efficient approach for multi-modal biometrics," International Journal of Pattern Recognition and Artificial Intelligence, vol. 25, no. 2, pp. 273-286, 2011, doi: 10.1142/S0218001411008555.

[31] M. Singh, R. Singh, and A. Ross, “A comprehensive overview of biometric fusion,” Information Fusion, vol. 52, pp. 187-205, 2019, doi: 10.1016/j.inffus. 2018.12.003.

[32] R. Ryu, S. Yeom, S. H. Kim, and D. Herbert, "Continuous Multimodal Biometric Authentication Schemes: A Systematic Review," IEEE Access, vol. 9. pp. 34541-34557, 2021, doi: 10.1109/ACCESS.2021.3061589.

[33] L. Kondapi, A. Rattani, and R. Derakhshani, "Cross-illumination Evaluation of Hand Crafted and Deep Features for Fusion of Selfie Face and Ocular Biometrics,” 2019, doi: 10.1109/HST47167.2019.9032976.

[34] G. Goswami, P. Mittal, A. Majumdar, M. Vatsa, and R. Singh, "Group sparse representation based classification for multi-feature multimodal biometrics," Information Fusion, vol. 32, pp. 3-12, 2016, doi: 10.1016/j.inffus.2015.06.007.

[35] D. Yaman, F. I. Eyiokur, and H. K. Ekenel, "Multimodal age and gender classification using ear and profile face images," in $I E E E$ Computer Society Conference on Computer Vision and Pattern Recognition Workshops, 2019, vol. 2019-June, pp. 2414-2421, doi: 10.1109/CVPRW.2019.00296.

[36] N. Poh and S. Bengio, "Database, protocols and tools for evaluating score-level fusion algorithms in biometric authentication," Pattern Recognition, vol. 39, no. 2, pp. 223-233, 2006, doi: 10.1016/j.patcog.2005.06.011.

[37] A. Nandhinipreetha and N. Radha, "Multimodal biometric template authentication of finger vein and signature using visual cryptography," 2016, doi: 10.1109/ICCCI.2016.7479963.

[38] R. M. Jomaa, H. Mathkour, Y. Bazi, and M. S. Islam, "End-to-end deep learning fusion of fingerprint and electrocardiogram signals for presentation attack detection," Sensors (Switzerland), vol. 20, no. 7, 2020, doi: 10.3390/s20072085.

[39] Y. Xin et al., "Multimodal feature-level fusion for biometrics identification system on IoMT Platform," IEEE Access, vol. 6, pp. 21418-21426, 2018, doi: 10.1109/ACCESS.2018.2815540.

[40] A. Tharwat, A. F. Ibrahim, and H. A. Ali, "Multimodal biometric authentication algorithm using ear and finger knuckle images," in Proceedings - ICCES 2012: 2012 International Conference on Computer Engineering and Systems, 2012, pp. 176-179, doi: 10.1109/ICCES. 2012.6408507.

\section{BIOGRAPHIES OF AUTHORS}

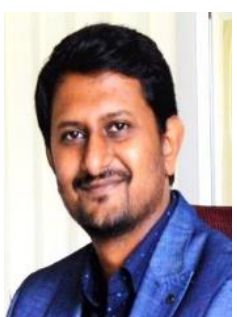

Arjun Benagatte Channegowda (D) SI SC P received a B.E. in CSE from VTU, Karnataka, India, and M.Tech. in Industrial Automation and Robotics from VTU Belagavi, Karnataka. India. Currently, he is pursuing a Ph.D. in Computer Science and Engineering at RIT research center affiliated to Visvesvaraya Technological University. He is currently working in ISE Department at RIT, Hassan as Assistant Professor and Head. He has 2 years of HCL industry and 11 years of teaching experience. He has published 2 scopus indexed papers and many conferences and international journals papers. His reseach interest in biometrics, image processing, and machine learning. He can be contacted at email: bc.arjun@gmail.com.

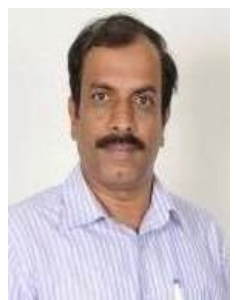

Dr. Hebbakavadi Nanjundaiah Prakash (D) $8 \mathrm{SC}$ P received a B.E. in ECE from Mysore University, and he received M.Tech. in Electronics Instrumentation from NIT Warangal, and received Ph.D. in Computer Science from Mysore University. He is currently working in CSE Department at RIT, Hassan as Professor and HOD. He has thiry years of teaching experience. He published IEEE transactions during his research work. He also published many papers in international journals and conferences. His research interest in biometrics, signature analysis, and artifical intelligence. He can be contacted at email: prakash_hn@yahoo.com. 